\title{
Relationship Between No/Slow Reflow Phenomenon in Acute Myocardial Infarction Patients After Percutaneous Coronary Intervention and Inflammatory Response
}

\author{
Binbin Wang ${ }^{1, *}$, Zhi Xiao², Nana Peng ${ }^{1}$ \\ ${ }^{1}$ Emergency Department, Binzhou People's Hospital, Binzhou City, P. R. China \\ ${ }^{2}$ Department of Nephrology, Binzhou People's Hospital, Binzhou City, P. R. China \\ Email address: \\ BinbinWang2019@163.com (Binbin Wang) \\ ${ }^{*}$ Corresponding author
}

To cite this article:

Binbin Wang, Zhi Xiao, Nana Peng. Relationship Between No/Slow Reflow Phenomenon in Acute Myocardial Infarction Patients After Percutaneous Coronary Intervention and Inflammatory Response. American Journal of Clinical and Experimental Medicine. Vol. 7, No. 4, 2019, pp. 93-96. doi: 10.11648/j.ajcem.20190704.13

Received: August 26, 2019; Accepted: September 24, 2019; Published: September 27, 2019

\begin{abstract}
Background: To explore the relationship between the occurrence of no/slow reflow phenomenon in acute myocardial infarction patients after undergoing percutaneous coronary intervention (PCI) and inflammatory response. Objective: Prospective study was conducted on 519 acute myocardial infarction patients undergoing PCI, in which 509 patients were followed up for one year. Method: The 76 cases with no/slow reflow phenomenon after PCI was the adverse reflow group. One hundred patients were randomly selected from the remaining 443 patients with the Excel random function table as the control group to avoid statistical deviation. Result: The inflammatory indicators, such as the total numbers of white blood cells and neutrophils, high-sensitivity C-reactive protein, mortality, adverse cardiac event. Conclusion: Inflammatory response is related to the occurrence of no/slow reflow phenomenon in acute myocardial infarction patients after PCI, and seriously affects their prognosis.
\end{abstract}

Keywords: Acute Myocardial Infarction, No/Slow Reflow, Direct PCI, Inflammatory Response

\section{Introduction}

Primary percutaneous coronary intervention (PCI) is the preferred method for the treatment of acute myocardial infarction (AMI). Restoring normal blood flow and myocardial perfusion is critical for reducing AMI cardiac events [1]. PCI not only opens the extra-epicardial coronary blood flow, but also achieve myocardial perfusion [2, 3]. Scholars have explored the pathogenetic mechanism of no reflow, but it remains controversial [4]; as for the long-term prognosis of patients who appear no/slow reflow directly after PCI, there is still lack of prospective clinical research data. In this study, analysis was made on the factors of the occurrence of no/slow reflow of AMI patients directly after PCI and the prognosis through long-term follow-up.

\section{Materials and Methods}

\subsection{Patients Receiving Direct PCI}

Five hundreds and nineteen AMI patients receiving direct PCI in the Heart Center of our hospital were selected and another 18 AMI patients whose direct PCI for infarction related artery (IRA) failed were not included in this study. Upon the informed consent of the family members, all AMI patients received coronary angiogram within $24 \mathrm{~h}$ after the disease and meanwhile, direct percutaneous coronary intervention was conducted to the fully-blocked culprit vessel, with the postoperative residual stenosis $<10 \%$. After PCI, the no/slow reflow was judged by two or above experienced interventional physicians, during PCI, the angiography indicated that the blood flow of thrombolysis in myocardial infarction (TIMI) was less than level 2, and thrombus, 
embolism, dissection aneurysm and spasm that might affect blood flow were excluded.

\subsection{Patients Selected}

Seventy-six no/slow reflow patients were selected as the experiment group. In order to avoid statistical deviation, at the end of follow up, 100 patients were randomly selected from all patients with postoperative immediate TIMI blood flow level 3 with the Excel random function table as the control group.

\subsection{Patients Drawn Blood}

All patients were drawn blood before undergoing PCI surgery to determine the high-sensitivity $\mathrm{C}$-reactive protein (hsCRP), blood lipid, insulin, platelet, white blood cells and neutrophils. The incidence of adverse events of all patients was recorded during hospitalization and after discharge, including death, heart failure, arrhythmias, angina pectoris, and myocardial reinfarction. After discharge, interventional outpatient follow-up was adopted, with a period of one year. There were 10 patients lost to be followed up, with the follow-up rate of $98.07 \%$. Medication for each patient before and after PCI fully complied with the Medication Guide of the current PCI surgery, including heparin, aspirin, clopidogrel and statin lipid-lowering agent, angiotensin converting enzyme inhibitor (ACEI) or angiotensin-receptor blockade (ARB) and B-blocker, etc. [5, 6].

\subsection{Patients Receive Echocardiography}

Each patient must receive echocardiography during hospitalization and at the end of follow-up, and the sonographer did not know the interventional treatment results which were reviewed by another sonographer. The left ventricular ejection fraction (LVEF) was measured with Simpson method, and two-dimensional method was used to measure the left ventricular end diastolic diameter (LVEDD) along the long axis section of parasternal left ventricle.

\subsection{Statistical Analysis}

All data were analyzed by SPSS 13.0. The numeration data were expressed as $\%$, and the measurement data were expressed as $(\mathrm{x} \pm \mathrm{s})$ by using $\mathrm{X}^{2}$ and $\mathrm{t}$ tests. $\mathrm{P}<0.05$ was considered statistically significant, and $\mathrm{P}<0.01$ was considered extremely significant.

\section{Results}

\subsection{Basic Information}

No statistically significant differences were found in the basic clinical data between the patients in the no/slow reflow group and those randomly selected from the control group, such as age, gender, length of coronary stent, the number of diseased vessels, blood lipid level, preoperative LVEF and LVEDD (Table 1).

Table 1. Basic information of the two groups.

\begin{tabular}{|c|c|c|c|}
\hline & & Control & No/slow reflow \\
\hline Total & & 100 & 76 \\
\hline Male/female (\%) & & $57 / 43(57 \% / 43 \%)$ & $41 / 35(53.95 \% / 46.05 \%)$ \\
\hline Age (years old) & & $60.91 \pm 8.47$ & $62.14 \pm 9.11$ \\
\hline \multirow[t]{2}{*}{ Stent length (mm) } & & $17.2 \pm 5.3$ & $16.7 \pm 6.1$ \\
\hline & 1 & $49(49 \%)$ & $36(47.37 \%)$ \\
\hline \multirow{2}{*}{ Number of diseased vessels } & 2 & $26(26 \%)$ & $22(28.95 \%)$ \\
\hline & 3 & $25(25 \%)$ & $18(23.68 \%)$ \\
\hline Total cholesterol $(\mathrm{mmol} / \mathrm{L})$ & & $5.6 \pm 1.2$ & $5.1 \pm 1.6$ \\
\hline LDL-C (mmol/L) & & $3.2 \pm 0.9$ & $3.4 \pm 1.1$ \\
\hline HDL-C (mmol/L) & & $1.3 \pm 0.3$ & $1.2 \pm 0.4$ \\
\hline LVEF (\%) & & $55.9 \pm 8.4$ & $56.2 \pm 9.7$ \\
\hline LVEDD (mm) & & $48.5 \pm 4.2$ & $46.8 \pm 6.4$ \\
\hline
\end{tabular}

\subsection{Risk Factors}

The analysis on the risk factors of the two groups showed that the hsCRP level, the total numbers of white blood cells and neutrophils of the no/slow reflow group were significantly higher than those of the control group, indicating that the inflammatory response of the former group is stronger than that of the latter. The prevalence of diabetes, insulin level and the platelet count of the no/slow reflow group were significantly higher than those of the control group $(\mathrm{P}<0.05$ or $\mathrm{P}<0.01$, Table 2 and Figure 1).

Table 2. Risk factors of the two groups.

\begin{tabular}{lllllll}
\hline $\mathbf{0}$ & Case No. & Diabetes & Insulin $(\boldsymbol{\mu I U} / \mathbf{m L})$ & $\begin{array}{l}\text { White blood cells } \\
\left(\times \mathbf{1 0}^{\mathbf{9}} / \mathbf{L}\right)\end{array}$ & $\begin{array}{l}\text { Neutrophils } \\
(\times \mathbf{1 0} / \mathbf{L})\end{array}$ & $\begin{array}{l}\text { Platelets } \\
(\times \mathbf{1 0} / \mathbf{L})\end{array}$ \\
\hline Control & 100 & $8(8 \%)$ & $16.2 \pm 5.4$ & $9.3 \pm 1.1$ & $6.2 \pm 1.8$ & $187.3 \pm 14.3$ \\
No/slow reflow & 76 & $14(18 \%)$ & $27.6 \pm 4.7^{*}$ & $12.2 \pm 1.8^{*}$ & $3.5 \pm 1.0$ & $9.1 \pm 1.4 * *$ \\
\hline
\end{tabular}

Compared with the control group, $* \mathrm{P}<0.05, * * \mathrm{P}<0.01$. 


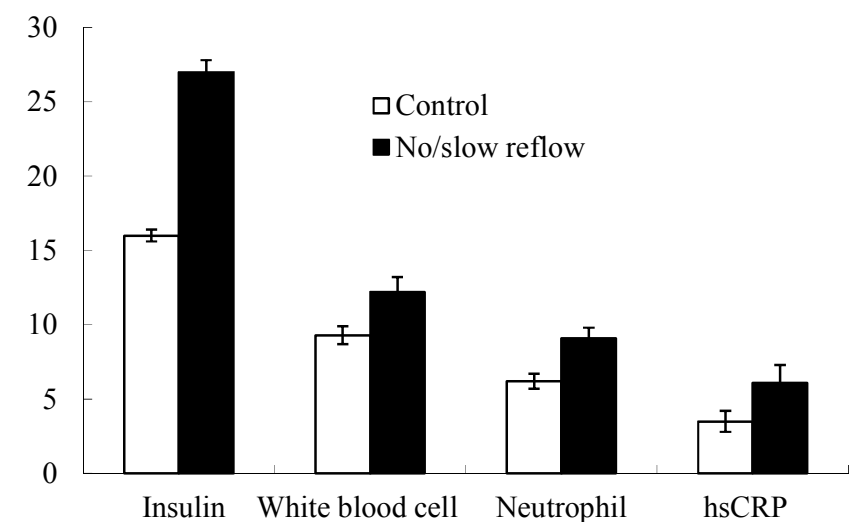

Figure 1. Risk factors of the two groups. Compared with the control group, $* P<0.05, * * P<0.01$.

\subsection{Adverse Events and Left Ventricular Structures and Functions}

The adverse events (including death, angina, heart failure, and recurrent infarction) in the no/slow reflow group during hospitalization and 12 months after discharge were significantly higher than those in the normal control group; LVEF in the no/slow-reflow group was significantly lower than that in the control group, and LVEDD was increased significantly compared with the control group (Figure 2).

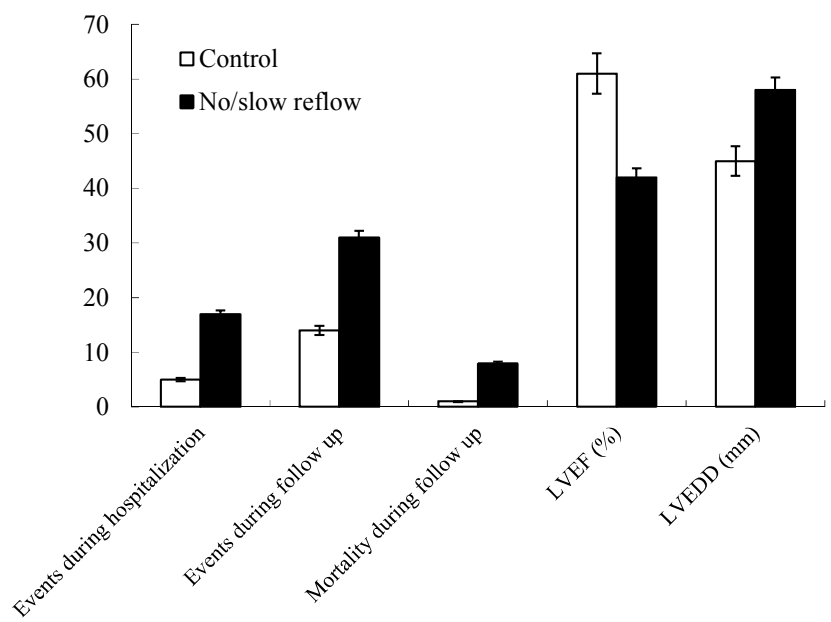

Figure 2. Adverse events and Left ventricular structures and functions during hospitalization and follow up.

\section{Discussion}

The goal of the treatment for AMI patients is to restore the blood perfusion of myocardial tissue in a rapid and complete way, to avoid the further progression of necrotic myocardial cells against cardiac pump failure. So PCI is the preferred therapy for the reconstruction of infarction related artery and restoration of reperfusion $[7,8]$. The occurrence of no/slow reflow phenomenon may exert a certain influence upon the prognosis of patients, but controversy remains over its mechanism of occurrence. Most scholars believe that it is related to micro-thromboembolism caused by reperfusion injury or white blood cells blocking blood capillary, but there is a lack of clinical evidence $[9,10]$. In this study, prospective observation was conducted on 519 patients with consecutive AMI undergoing PCI treatment, of which 76 patients suffered from no reflow after PCI, with the incidence rate of $14.7 \%$, being consistent with other reports.

The results of this study showed that the levels of white blood cells, neutrophils and hsCRP in the no/slow reflow group were significantly higher than those in the control group, suggesting that inflammatory response may play an important role in the occurrence of no/slow reflow which especially tends to appear in patients with relatively strong inflammatory response. Healey et al. found that the number of white blood cells of AMI patients with no reflow after PCI was higher than that of patients with normal reflow [11]. While in this study, the total numbers of white blood cells and granulocytes of patients in the no/slow reflow group were higher than those in the normal reflow group, and the platelet count and hsCRP levels were also significantly elevated, indicating that the high intensity of inflammatory response and blood viscosity is one of the conditions for the occurrence of no reflow, which perfect the above inference in a better way. In the no/slow reflow group, the ratio of diabetes and insulin level were significantly higher than those in the control group, which may be related to the reason that high blood sugar increases the expression of adhesion molecules, resulting in the expansion of white blood cells in capillary blockage, high blood sugar promoting thrombosis and increased reperfusion injury. At the same time, high insulin level can also lead to vascular endothelial dysfunction for microvascular dysfunction $[12,13]$. Therefore, diabetes or hyperinsulinemia is also one of the reasons for the occurrence of no reflow phenomenon. Berstad et al. also observed through PCI treatment for 146 AMI patients that the ratio of hyperglycemia in the no reflow group was significantly higher than that in the control group [14].

Although coronary artery dominating the core of the necrotic area is revascularized, microvascular injury and secondary obstruction still limit the reperfusion of infarct core. Under electron microscope, capillary endothelial cell swelling, intravascular neutrophil obstruction and thromboembolism can be found, which might affect the prognosis of patients in clinical practice. The increased extent of microvascular obstruction is related to the changes of myocardial stress in and around the infarct area. If the microvascular obstruction of the infarct area is larger than $35 \%$, myocardial passive elongation of this area and the thickness of the surrounding musculus cardiacus will be reduce significantly, causing poor reconstruction of no-reflow area $[15,16]$. Srikanth et al. analyzed the clinical significance and prognosis of the left ventricular function in patients with coronary no reflow after emergency PCI, and the results showed that LVEF of the patients was decreased, while LVEDV and LVESV were increased, with left ventricular dilatation and remodeling; and there was a high incidence of malignant arrhythmia, cardiogenic death and other cardiovascular events [17]. Coronary no reflow is an independent predictive factor for left ventricular dysfunction and cardiovascular events of myocardial infarction patients after emergency PCI, and 
enhanced inflammatory response may be one of the main conditions of the occurrence of no reflow phenomenon. The data also showed that the adverse events in the no/slow reflow group during hospitalization and 12 months after discharge was higher than that of the control group, and LVEF in the former group was significantly lower compared with the latter group before and after follow-up, and LVEDD was increased. The mortality rate of the no reflow group was higher than that of the normal blood flow group, indicating that the no/slow reflow phenomenon in AMI patients after PCI is a strong predictive factor of poor prognosis.

\section{Conclusion}

No/slow reflow, as a serious complication after PCI, can significantly increase the adverse events during hospitalization and follow-up. In addition to the influence of diabetes and insulin level, its occurrence is also related to the intensity of inflammatory response. No/slow reflow after PCI is a predictive factor of continuous myocardial ischemia, ventricular remodeling and cardiac functional recovery disturbance, as well as a sign of myocardial and microvascular injury.

\section{References}

[1] Ambrose JA. Editorial: Managing Coronary Thrombus in the Cath Lab During PCI. Curr Cardiol Rev, 2018, 8: 200-1.

[2] Lazzeri $\mathrm{C}$, Valente $\mathrm{S}$, Chiostri $\mathrm{M}$, Attanà $\mathrm{P}$, Mattesini $\mathrm{A}$, Dini CS, Gensini GF. Comorbidities in stemi patients submitted to primary PCI: Temporal trends and impact on mortality: A 6-year single center experience. Int J Cardiol, 2019, 11 (8): 256-31.

[3] Balghith MA. High Bolus Tirofiban vs Abciximab in Acute STEMI Patients Undergoing Primary PCI - The Tamip Study. Heart Views, 2018, 13 (3): 85-90.

[4] Palmerini T, Genereux P, Caixeta A, Cristea E, Lansky A, Mehran R, Della Riva D, Fahy M, Xu K, Stone GW. A New Score for Risk Stratification of Patients With Acute Coronary Syndromes Undergoing Percutaneous Coronary Intervention: The ACUITY-PCI (Acute Catheterization and Urgent Intervention Triage Strategy-Percutaneous Coronary Intervention) Risk Score. JACC Cardiovasc Interv, 2019, 5 (11): 1108-16.

[5] Cohn JN. Fractional flow reserve-guided PCI reduced urgent revascularization at 7 months in coronary artery disease. Ann Intern Med, 157 (10): 5-9.
[6] Huang RL, Thomassee EJ, Park JY, Scott C, Maron DJ, Fredi JL. Clinical Pathway: Helicopter Scene STEMI Protocol to Facilitate Long-Distance Transfer for Primary PCI. Crit Pathw Cardiol, 2019, 11 (4): 193-8.

[7] Jhaveri RR, Reynolds HR, Katz SD, Jeger R, Zinka E, Forman SA, Lamas GA, Hochman JS. Heart Failure in Post-MI Patients With Persistent IRA Occlusion: Prevalence, Risk Factors, and the Long-Term Effect of PCI in the Occluded Artery Trial (OAT). J Card Fail, 2018, 18 (11): 813-21.

[8] Giordano V, Grandjean JG. There is always hope after PCI and stenting. J Cardiovasc Med (Hagerstown), 2019, 13 (11): 766-8.

[9] Knot J, Kala P, Rokyta R, Stasek J, Kuzmanov B, Hlinomaz O, Bĕlohlavek J, Rohac FP, Petr R, Bilkova D, Djambazov S, Grigorov M, Widimsky P. Comparison of outcomes in ST-segment depression and ST-segment elevation myocardial infarction patients treated with emergency PCI: data from a multicentre registry. Cardiovasc J Afr, 2018, 23 (9): 495-500.

[10] Kherada N, Mehran R. Impending savior: Impella 2.5 circulatory support system in high-risk PCI. Catheter Cardiovasc Interv, 2017, 80 (5): 726-7.

[11] Healey JS. Trifecta or Triple Threat? The Challenge of Post-PCI Management in Patients Receiving Chronic Oral Anticoagulant Therapy. Can J Cardiol, 2019, 11 (2): 641-5.

[12] Moscucci M. Public reporting of PCI outcomes and quality of care: one step forward and new questions raised. JAMA, 2018, 308 (14): 1478-9.

[13] Chakrabarti AK, Gibson CM. Optimal selection of STEMI treatment strategies in the current era: benefit of transferring STEMI patients for PCI compared with administration of onsite fibrinolytic therapy. Curr Opin Cardiol, 2019, 27 (6): $651-4$.

[14] Berstad MB, Weyergang A, Berg K. Photochemical internalization (PCI) of HER2-targeted toxins: Synergy is dependent on the treatment sequence. Biochim Biophys Acta, 2017, 1820 (12): 1849-58.

[15] Jerónimo Sousa P, Campante Teles R, Brito J, Abecasis J, de Araújo Gonçalves P, Calé R, Leal S, Dourado R, Raposo L, Silva A, Almeida M, Mendes M. Primary PCI in ST-elevation myocardial infarction: Mode of referral and time to PCI. Rev Port Cardiol, 2017, 31 (10): 641-646.

[16] Meier P, Froehlich GM, Yellon DM, Hausenloy DJ. Predicting peri-procedural myocardial infarction during PCI. Heart, 2018, 98 (20): 1471-2.

[17] Srikanth S, Ambrose JA. Pathophysiology of Coronary Thrombus Formation and Adverse Consequences of Thrombus During PCI. Curr Cardiol Rev, 2018, 8 (3): 168-76. 